\title{
Thermally Stimulated Discharge of Polymer Electrets*
}

\author{
J. van Turnhout
}

Centraal Laboratorium TNO Delft, Holland.

(Received October 3, 1970)

\begin{abstract}
A special form of depolarization of polymer electrets is described: the thermally stimulated discharge (TSD). After reviewing its principles and exposing its mathematical analysis, results are presented which show that TSD is a powerful method to gain insight into the molecular mechanisms of the electret effect. In fact, TSD reveals all low-frequency molecular motions. It enables one to determine, e.g., the glass-rubber transition of polymers. Its merits are compared with those of isothermal dielectric, and mechanical methods of investigation.
\end{abstract}

KEY WORDS Electrets / Depolarization / Non-Isothermal Dielectric

Theory/Thermal Analysis/Relaxation/Poly(methyl methacrylate)/

Copolymers/Poly(ethylene terephthalate)/Glass-Transition/

It is well known that many dielectrics do not respond instantaneously to voltage changes. In electrets, the analogues of magnets, this delay is particularlypronounced; they retain their charge "permanently" after having been subjected to a temperature-field treatment.

Electrets were discovered by Eguchi in 1920. Since that time they were studied by several workers, of whom Gross, in particular, made important contributions to their understanding. Most of this early work is described in the literature. $^{1-5}$ For more recent work we refer to Baxt and Perlman ${ }^{6}$.

The potentialities of electrets did not seem promising, until Sessler and West ${ }^{7}$ successfully developed a foil-electret microphone. This microphone was put into commercial production by SONY (Japan) a year ago. Further applications are to be expected, now that polymer electrets have become available with decay times of many years.

Recently, considerable progress has also been made in the understanding of the charging and discharging mechanisms, both of which are complicated. First of all, the charging is a non-isothermal process which, in general, involves two chargings of opposite sign. In addition, the charging phenomena are heavily masked by conduction. The latter is not

* Part of this paper was presented at the 1st International Conference on Static Electricity, Vienna, Austria., May 4-6, 1970. observed during a discharge with short-circuited electrodes. It is therefore easier to study the discharge, in which the same molecular phenomena occur as during charging. In order to perform these experiments in a reasonably short time, the discharge has to be thermally stimulated (TSD).

Although non-isothermal discharge has been investigated since $1936^{8}$; it was not until 1966 that its theoretical basis could be given'. Since then the theory has been considerably extended $^{10-13}$, while progress in its experimental use is being made in several laboratories ${ }^{12-19}$.

As we will show in this paper TSD is not only of importance for electrets. It is also able to elucidate the low-frequency behaviour of dielectrics, about which little is as yet known. Moreover, the release of frozen-in mechanical stresses, which cause undesirable dimension instability of plastics, bears some relation to TSD.

\section{THE CHARGING OF ELECTRETS}

The formation of an electret is illustrated in Figure 1. The polymer is heated to above its glass-rubber transition, $T_{g}$. At time $t_{0}$, an electric field is applied, which causes an alignment of dipoles and a drift of real charges. At time $t_{f}$, the polymer is cooled, whereby the main polymer chains are immobilized. Consequently, most of the dipoles and charges are frozen in. As a result they do not respond 


\section{J. van TuRnhout}

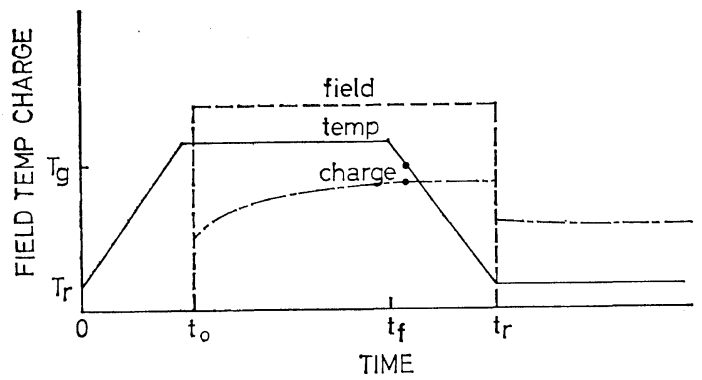

Figure 1. Scheme of the formation of polymer electrets.

when the field is switched off at $t_{r}$; only the instantaneous polarization disappears. Therefore, when the electret is removed from the formation unit, the charge is largely retained; in polymers of low conductivity having a high $T_{g}$ even for years.

The contact between electrodes and polymer often is far from perfect (Figure 2), in which event besides the internal charging, an external charging may occur. At high field strengths breakdown takes place in the air inclusions, so that ions* are injected into the polymer. In view of their polarity with respect to the electrodes, the two opposite charges are called hetero charge and homo charge. Homocharging is experimentally detected by ammeter $\mathrm{A}$ as noise on the charging current.

One of our aims was to gain some insight into the molecular mechanism involved. Moreover, we sought to relate the formation conditions with the charge created. However, the number of pertinent variables is discouragingly large. Therefore, we eliminated homocharging by using evaporated silver electrodes. To bring out the unique effect of the polymer, we carried out our TSD measurements on electrets formed under identical conditions, except for the temperature, which was adjusted to above $T_{g}$. For the field strength we chose $50 \mathrm{kV} / \mathrm{cm}$, for the formation time 1.5 hours, and for the heating and cooling rate $2^{\circ} \mathrm{C} / \mathrm{min}$. The samples were $2-\mathrm{mm}$ thick.

\section{THEORY OF THERMALLY STIMULATED DISCHARGE}

As we have remarked, a hetero- and homocharge are, generally, formed, which together consist of a polarization, a volume charge and a surface charge. It is of major interest to know the various contributions. They cannot be distinguished by charge measurements alone, only during discharge do they manifest in different ways ${ }^{20}$. As the decay proceeds slowly at room temperature, it is advisable to stimulate the discharge by heating.

The use of TSD dates back to Frei and Groetzinger ${ }^{8}$. Later, it was employed by $\mathrm{Gross}^{21}$, Gubkin $^{22}$ and coworkers, who programmed the temperature arbitrarily, being interested only in

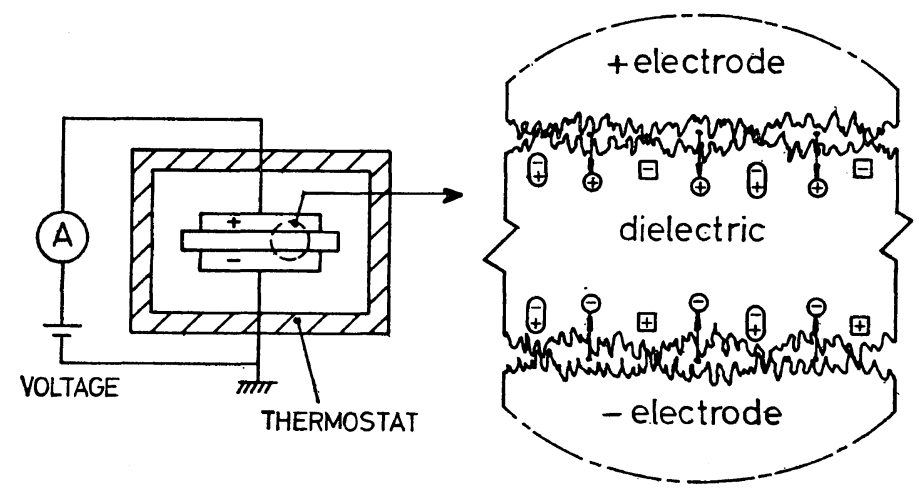

Figure 2. Illustration of the injection of homocharges $\oplus$. The internal heterocharging by dipole orientation $\Theta$ and ion migration $\boxminus$ is also shown.

* The injection of electrons is unlikely, they are too quickly attached to air molecules. 
the ultimate charge. As was already pointed out by von Altheim, ${ }^{23}$ charge release is controlled by molecular movements, which depend markedly on temperature. For this reason we raised the temperature linearly ${ }^{12}$ as Bucci, et al., ${ }^{9}$ advanced for inorganic dielectrics. Miller, et al. ${ }^{15}$ also used this new technique, and in several laboratories encouraging progress is being made in its practice.

Recently, interesting results were obtained with it by Takamatsu, et al., ${ }^{17}$ who studied $\mathrm{PE}, \mathrm{PVF}_{2}$, and PTFE; ${ }^{*}$ by Lilly, et al., ${ }^{18}$ who gave several results of PET at different fields and temperatures, and by Creswell and Perlman $^{13,14}$ who carried out theoretical and experimental investigations on corona-charged PET. Actually, the method can be employed for all kind of materials, including semiconductors. $^{24,25}$ It was applied by Nedetzka, et al. ${ }^{19}$ (who were not aware of earlier work on TSD), to reveal the dielectric properties of hemoglobin.

Bucci, et al., put forward a theory of TSD as well. This theory has lately been generalized by Gross $^{10,11}$ and extended by the author to include polymers. ${ }^{12}$ So far the theory was was mainly concerned with dipole reorientation in metallized samples. A theory about the TSD of space chages is being developed $;^{13,19,24,25}$ in this paper some of its aspects will be discussed. Furthermore, TSD using air gaps, to study homocharge decay, will be treated.

\section{TSD of Metallized Electrets}

First let us consider dipoles to be present. The charging and discharging due to permanent dipoles are usually described by the celebrated time superposition principle (TSP). In our case the processes are non-isothermal, thus the TSP has to be enlarged into a temperature-time superposition principle. ${ }^{10,12,26}$

In our experiments heating rates were constant, so starting TSD just after short-circuiting at room temperature, the charging and discharging scheme of Figure 3 results. We supposed the internal field during TSD to be zero, neglecting the voltage across the ammeter for measuring the discharge current.

According to TSP, if the formation field is

* Abbreviations of polymers are explained in Table II.

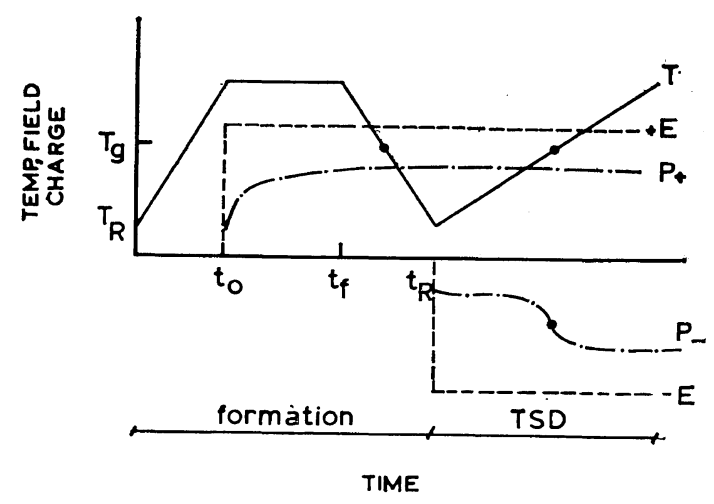

Figure 3. Illustration of the temperature-time superposition during formation and TSD.

$+E$, the polymer will react to a field $-E$ during TSD. Heating will accelerate this response. Assuming a single dipole relaxation to be active, the polarization $P$ - created, is given by

$$
d P / d t=-\alpha P+\varepsilon_{0}\left(\varepsilon_{s}-\varepsilon_{\infty}\right) \alpha E
$$

In this well-known Debye equation, $t$ denotes the time, $\alpha$ the relaxation frequency and $\varepsilon_{s}, \varepsilon_{\infty}$ the dielectric constant at low and high frequencies, respectively. As a result of the heating $\alpha$ and the relaxation strength $\varepsilon_{s}-\varepsilon_{\infty}$ vary. Often, $\varepsilon_{s}-\varepsilon_{\infty}$ is proportional to $1 / T$, while $\alpha$ varies exponentially with it. For simplicity we take $\varepsilon_{s}-\varepsilon_{\infty}$ to be constant.

In polymers various dipole relaxations are possible, and the temperature dependence of $\alpha$ is not unique. For relaxations due to local motions of polar side groups, an Arrhenius equation is appropriate

$$
\alpha=\alpha_{0} \exp (-A / k T)
$$

where $\alpha_{0}$ is approximately constant, $A$ is the activation energy, $k$ Boltzmann's constant and $T$ the absolute temperature. Moreover, polar groups may move cooperatively with the main chains. Since the motions of bulky main chain segments require some unoccupied or free volume, this relaxation shift according to the WLF equation*

$$
\alpha=\alpha_{g} \exp c_{1}\left(T-T_{g}\right)\left(c_{2}+T-T_{g}\right)^{-1}
$$

where $\alpha_{g} \simeq 7 \times 10^{-3} \mathrm{sec}^{-1}, c_{1}=40$ and $c_{2}=52 \mathrm{deg}{ }^{\circ} \mathrm{C}$

* WLF stands for Williams, Landel, and Ferry. 


\section{J. van TURNHOUT}

for amorphous polymers. $T_{g}$ represents the glasstransition temperature, at which the conformational rearrangements of the main chains are initiated.

The WLF equation only holds above $T_{g}$. Below $T_{g}$, similar to the polarization, a part of the unoccupied volume is frozen in. To describe this non-equibrium behaviour, Rush $^{27}$ introduced an "effective" temperature to replace $T_{g}$. Earlier, Macedo et al. ${ }^{28}$ suggested use of a hybrid of eq 2 and 3

$$
\begin{aligned}
\alpha= & \alpha_{g} \exp \left\{c_{1}\left(T-T_{g}\right)\left(c_{2}+T-T_{g}\right)^{-1}\right. \\
& \left.+A\left(T-T_{g}\right) / k T T_{g}\right\}
\end{aligned}
$$

Eq (2)-(4) can be written in "shorthand" as

$$
\alpha=\alpha_{r} a_{T}
$$

where $a_{T}$ is one of the temperature shifts and $\alpha_{r}$ the corresponding prefactor.

The acceleration of the polarization having been specified, eq 1 can be integrated. Starting at time $t_{R}$, we have

$$
P_{-}=\varepsilon_{0}\left(\varepsilon_{s}-\varepsilon_{\infty}\right) E\left\{1-\exp \left(-\alpha_{r} \int_{t_{R}}^{t} a_{T} \mathrm{~d} t\right)\right\}
$$

The inverse heating rate, $s=\mathrm{d} t / \mathrm{d} T$, being constant, we may write for the so-called reduced time

$$
\xi=\int_{t_{R}}^{t} a_{T} \mathrm{~d} t=s \int_{T_{R}}^{T} a_{T} \mathrm{~d} T
$$

Interestingly enough, eq 6 does not differ much from the isothermal case. The actual time has merely been replaced by a reduced time.

The charging due to field $+E$ is described in the same way. It is easily verified that a polarization

$$
\begin{aligned}
P_{+}= & \varepsilon_{0}\left(\varepsilon_{s}-\varepsilon_{\infty}\right) E\left\{1-\exp \left(-\alpha_{f} t_{f}-\alpha_{r}\right.\right. \\
& \left.\left.\times \int_{t_{f}}^{t_{R}} a_{T} \mathrm{~d} t-\alpha_{r} \int_{t_{R}}^{t} a_{T} \mathrm{~d} t\right)\right\}
\end{aligned}
$$

is formed. Subscripts $f, R$ refer to the formation temperature $T_{f}$, and room temperature $T_{R}$, respectively.

Finally, by subtracting eq 6 from 8 we obtain for the charge persisting after a TSD to temperature $T$

$$
\Pi=F_{\varepsilon_{0}}\left(\varepsilon_{s}-\varepsilon_{\infty}\right) E \exp \left(-\alpha_{r} \xi\right)
$$

where constant $F$ depends on the conditions of formation

$$
F=1-\exp \left(-\alpha_{f} t_{f}-\alpha_{r} \int_{t_{f}}^{t_{R}} a_{T} \mathrm{~d} t\right) .
$$

Normally, one charges the polymer completely by choosing $T_{f}$ and $t_{f}$ properly, in this case $F=$ 1. Considering the normalized value $\Pi / \varepsilon_{0} E$, charge release depends only on polymeric constants like: $\varepsilon_{s}-\varepsilon_{\infty}, \alpha_{r}$ and $a_{T}$. Obviously, when the discharge temperature exceeds $T_{f}$ the frozenin charge becomes exhausted.

The normalized current density during TSD is found by differentiating eq 9 with respect to time

$$
j / \varepsilon_{0} E=\alpha \Pi / \varepsilon_{0} E
$$

To see what kind of TSD thermograms can be expected, we substitute in eq 9 and 10 an Arrhenius shift. Unfortunately, the integral

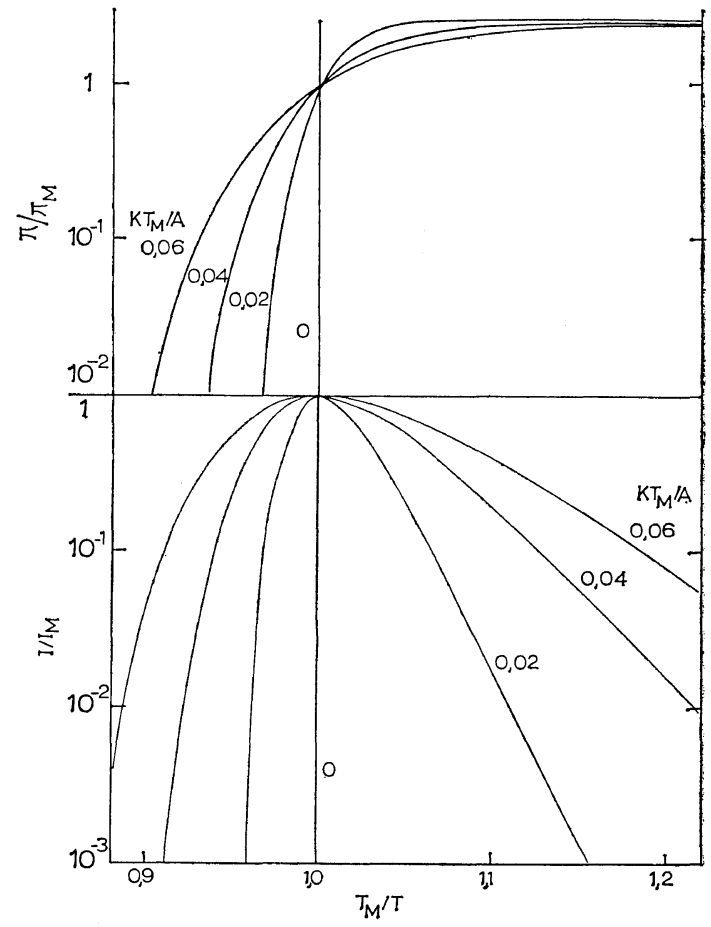

Figure 4. Calculated TSD charge and current thermograms. The trend is shown for three activation energies. All units are reduced to values at the current maximum. 
occurring in $\xi$ is not elementary. However, by partial integration it can be expressed in the exponential integral $E_{1}(x)$. Writing $x$ for $A / k T$ we obtain, by substitution of the asymptotic expansion of $E_{1}(x)$ :

$$
\begin{aligned}
& \int_{0}^{T} \exp (-A / k T) \mathrm{d} k T / A \\
& \quad=\frac{e^{-x}}{x}-E_{1}(x)=\frac{e^{-x}}{x^{2}}\left(1-\frac{2 !}{x}+\frac{3 !}{x^{2}}-\cdots\right)
\end{aligned}
$$

For most polymers $x>40$, so the series converges fast. The rational functions given by Hastings ${ }^{29}$ are also convenient for evaluating $E_{1}(x)$.

The charge persisting and the current released are plotted vs. 1/T in Figure 4. The charge decreases to zero in a narrow temperature range, especially for high activation energies. At temperature $T_{m}$, where the charge decreases most rapidly, the current reaches a maximum. It rises more slowly with increasing temperature than it falls, giving the graphs an asymmetric appearance. According to the WLF equation the transition is centered on $T_{g}$ for the main chain relaxation.

The typical structure of polymers admits of different conformations of the dipoles. Therefore, the polarization seldom relaxes at one frequency. A distribution over a number of discrete, or even a continuous range of relaxation frequencies is more likely.

For this case we obtain a set of simultaneous differential equations which for isothermal conditions, lead to a polarization

$$
P_{-}=\varepsilon_{0}\left(\varepsilon_{s}-\varepsilon_{\infty}\right) E \int_{0}^{\infty} f(\alpha)\{1-\exp (-\alpha t)\} \mathrm{d} \alpha
$$

where $f(\alpha)$ is the normalized distribution function of the relaxation strength, thus $\int_{0}^{\infty} f(\alpha) \mathrm{d} \alpha=1$. For non-isothermal conditions each subrelaxation may possess its own frequency shift $a_{T}$. However, for the same relaxation mechanism, e.g., the rearrangements of the main chains, this seems unlikely. There is much experimental evidence that several polymers behave thermorheologically simple (Staverman, et al. ${ }^{30}$ ), implying that all partaking relaxations have the the same temperature shift $a_{T}$. The distribution function then is temperature-independent and for the non-isothermal case we simply get

$$
P_{-}=\varepsilon_{0}\left(\varepsilon_{s}-\varepsilon_{0}\right) E \int_{0}^{\infty} f(\alpha)\left\{1-\exp \left(-\alpha_{r} \int_{t_{R}}^{t} a_{T} \mathrm{~d} t\right)\right\} \mathrm{d} t
$$

so that for the charge and current during TSD we can derive

$$
\begin{aligned}
& \Pi / \varepsilon_{0} E=\left(\varepsilon_{s}-\varepsilon_{\infty}\right) \int_{0}^{\infty} F f(\alpha) \exp \left(-\alpha_{r} \xi\right) \mathrm{d} \alpha \\
& j / \varepsilon_{0} E=\left(\varepsilon_{s}-\varepsilon_{\infty}\right) \int_{0}^{\infty} \alpha F f(\alpha) \exp \left(-\alpha_{r} \xi\right) \mathrm{d} \alpha
\end{aligned}
$$

$F$ has been written in the integrals, because it is a function of $\alpha$. As a result the original distribution function $f(\alpha)$ is modified to an effective one $F f(\alpha)$, which depends on the formation parameters. Therefore, in contrast to the reduced TSD results of an undistributed polarization as shown in Figure 4, those of a distributed one will depend on the formation conditions, unless one makes $F=1$, by charging the polymer completely.

To apply eq 14 and 15, the distribution function $f(\alpha)$ must be known. According to eq 2 a distribution of relaxation frequencies may arise from a distribution of activation energies or from a distribution of pre-exponential factors. Recently Gross $^{10}$ considered an exponential distribution of activation energies. For these low frequency phenomena a Voglis or Wagner distribution may be equally valid. For isothermal discharges both lead to the well-known von Schweidler relation $j \infty t^{-n}$. Again supposing that these distributions only shift, the resulting TSD is easily found. ${ }^{12}$ A still better way is not to prescribe any particular distribution, but to calculate $f(\alpha)$ from the experimental data (see Evaluation of TSD data).

Our investigations (see DISCUSSION OF EXPERIMENTAL RESULTS) reveal that, besides dipoles, space charges are frozen in. Let us therefore turn to the TSD of space charges. In general, these tend to pile up near the boundaries of different materials (MaxwellWagner effect). In homogeneous polymers they accumulate near the electrodes, in heterogeneous polymers on the grain interfaces.

Real charges may decay by Ohmic conduction of the polymer, or they may vanish by drifting 


\section{J. van TuRnhout}

and diffusion. If the electrodes are nonblocking, Ohmic discharge produces no external current. ${ }^{20}$ The same holds for decay by diffusion due to concentration gradients. Thus, only the drift of the released charges can be observed, provided that their transit time is shorter than the Ohmic relaxation time, for otherwise the charges are neutralized before they drift away. It was found to be unnecessary to control charge drift by applying a small voltage, as is done in photoconductive-glow curve experiments. ${ }^{31,32}$

We describe the drift due to the internal field phenomenologically as Lindmayer ${ }^{23}$ did, in another context, for the isothermal case. The short-circuit causes the field to become zero at some point $x^{*}$. During TSD, $x^{*}$ will shift, giving rise to a displacement current

$$
j=(p-n)^{*} \mathrm{~d} x^{*} / \mathrm{d} t
$$

where $(p-n)^{*}$ is the space-charge density at $x^{*}$.

Either a hetero- or homocurrent is possible, depending on the sign of $\mathrm{d} x^{*} / \mathrm{d} t$. For reasons of symmetry a heterodrift to the middle of the sample is more likely. This drift releases a charge of

$$
\sigma=\int_{x_{0}}^{l / 2}(p-n)^{*} \mathrm{~d} x^{*}
$$

if $x^{*}$ starts at $x_{0}$. The charge emerging is smaller than the charge initially present, because charges flow away to both electrodes. For electrons distributed as $n \infty x^{-1 / 2}$, Lindmayer estimates a fraction of $6 \%$ to be measurable. In order to specify the thermograms, the relation of $(p-n)^{*}$ and $x^{*}$ to temperature must be known. So far we have been able to solve this problem analytically for simple distributions only. ${ }^{34}$

Lindmayer pointed out another kind of discharge, the temperature dependence of which can be examined more easily. In fact, the release of electrons from their traps may take more time than their transit drift. The escape mechanism then governs the discharge. Lindmayer intimated that for slow retrapping an external current appears only when the traps are distributed in energy, say, uniformly between $E_{1}$ and $E_{2}$. He argued that the deep levels are filled from source to sink. Thus, $E_{1}$ is a function of distance. Denoting the number of charge-filled traps by $N$, and the escape frequency by $\nu=\nu_{0} \exp (-E / k T)$, where $E$ is the depth of the traps, he found for the isothermal current density from a unit volume

$$
\Delta j=e \mathrm{~d} N / \mathrm{d} t=e N_{0} \nu_{0} \int_{E_{1}}^{E_{2}} e^{-E / k T} \exp (-\nu t) \mathrm{d} E / k T,
$$

where $e$ is the electron charge.

In adapting eq 18 to TSD, we must bear in mind that the escape is thermally activated. Accordingly, we obtain

$\Delta j=e N_{0} \nu_{0} \int_{E_{1}}^{E_{2}} e^{-E / k T} \exp \left(-S \nu_{0} \int_{T_{R}}^{T} e^{-E / k T} \mathrm{~d} T\right) \mathrm{d} E / k T$

Although the external current is closely related to $\Delta j$, an explicit relation cannot easily be given. However, if one assumes that the time dependence of $j$ and $\Delta j$ are equal, eq 19 can be numerically integrated. The thermograms obtained happen to be broader than those of Figure 4, since eq 19 corresponds to a polarization with a uniformly distributed activation energy.

An interesting expression for a specific homopolar space-charge distribution was recently given by Creswell, et al. ${ }^{13}$ They considered a slab charged uniformly by electrons injected into a thin surface layer of thickness $\delta$. Instead of eq 16 they used for the external current due to drifting charges

$$
j=(\mu / l) \int_{0}^{l} \rho_{f} E \mathrm{~d} x
$$

where $\rho_{f}$ is the density of free electrons and $\mu$ their mobility. Eq 20 can be derived from Poisson's equation and from the equation of continuity. By substitution of

$$
E=-\left(\rho \delta / \varepsilon_{0} \varepsilon\right)(1-\delta / 2 l)+\left(1 / \varepsilon_{0} \varepsilon\right) \int_{0}^{x} \rho \mathrm{d} x
$$

eq 20 can be written as

$$
j=\left(\mu \delta^{2} / 2 \varepsilon_{0} \varepsilon l\right)(1-\delta / l) \rho_{f} \rho
$$

where $\rho$ denotes the total density of free and trapped electrons.

In eq $21 \rho$ and $\rho_{f}$ will be functions of time, whereas $\delta$ remains almost constant for this typical 
trapped-charge distribution. Creswell, et al.,
specified the time-temperature dependence of $\rho$ and $\rho_{f}$ by invoking the trap kinetics. For slow retrapping and assuming $\rho_{f}<\rho$, and $\delta<l$ they finally obtained

$$
\begin{aligned}
j= & \left(\mu \rho_{0}{ }^{2} \delta^{2} v / 2 \varepsilon_{0} \varepsilon l \nu_{R}\right) \\
& \times \exp \left\{-2 s \nu_{0} \int_{T_{R}}^{T} \exp (-E / k T) \mathrm{d} T\right\}
\end{aligned}
$$

where $\nu_{R}$ is the recombination frequency and $\nu$ the thermally stimulated escape frequency. ${ }^{*}$

This equation again shows a trend similar to Figure 4 , but because of the factor 2 in the exponential, the peak will be more pronounced. For the use of eq 22 to evaluate the trap kinetics, we refer to Creswell and Perlman. ${ }^{13}$ For other distributions $\delta$ is not constant and the differential equations have to be solved numerically, as Monteith, et al. ${ }^{35}$ have done for the isothermal case.

Earlier Zhdan, et al. ${ }^{24}$ and Zolotaryov, et $a l .{ }^{25}$ published similar theories. The former considered a field-effect structure, while the latter studied the TSD due to barrier polarization with blocking electrodes. Clearly, existing theories based on trap release are of limited application. No general theory has been published as yet. Moreover, in using a theory based on trap release we must remember that for amorphous polymers the existence of energy bands is still questionable.

Although diffusion produces no current with nonblocking electrodes, it does so with partly blocking ones. An isothermal theory about this discharge process was published by Jaffé, et al ${ }^{36}$ In order to solve the nonlinear differential equations involved, they neglected the formation and recombination of charges and linearized the internal field by putting it zero.

$$
j=2 \mu c E \sum_{n=0}^{\infty} G_{n} \exp \left(-z_{2 n+1}^{2} \theta\right)
$$

where $\mu$ is the mobility and $2 c$ the total equilibrium density of positive and negative charge carriers, $E$ the formation field and $\theta$ the normalized time. The constants $G_{n}$ depend on the normalized formation voltage $v$, the normalized blocking factor $\rho$, and the eigenvalues

* Note that in a band-structure model, $\mu$ and $\nu_{R}$ are assumed to be nearly temperature independent. $z_{2 n+1}$.

$$
8 G_{n} / v^{2}=\left\{\left(1+v^{-2} z_{2 n+1}^{2}\right)\left(1+\left(\rho^{2}+2 \rho\right) z_{2 n+1}^{-2}\right)\right\}^{-1}
$$

The normalized quantities $v, \rho$, and $\theta$ are defined by $v=\mu V / D, \rho=\xi l / D$ and $\theta=D t / l^{2}$, where $V$ is the formation voltage, $D$ the diffusion constant, $\xi$ the blocking factor, $l$ the thickness of the sample and $t$ the time. The eigenvalues $z_{2 n+1}$ follow from

$$
\tan z=2 \rho z\left(z^{2}-\rho^{2}\right)^{-1}
$$

As the eigenvalues differ by a factor of about $2 \pi$, the series of eq 23 converges fast; for longer times only the first term remains.

During TSD particularly $\mu$ and $D$ will increase. According to Einstein's relation, $\mu / D=$ $e / k T$, both quantities shift by about the same factor $b_{T}$. Frequently, Arrhenius' relation eq 2 holds for $b_{T}$. Putting $\mu=\mu_{r} b_{T}, \quad \eta=\int_{0}^{t} b_{T} \mathrm{~d} t$ and ignoring the second and higher terms eq 23 becomes

$$
j=2 \mu c E G_{0} \exp \left(-z_{1}^{2} \theta_{r} \eta\right)
$$

Note that diffusion leads to thermograms similar to those of dipole reorientation, but differing from the latter in that the fielddependence may no longer be linear. ${ }^{36}$

Besides amorphous polymers we investigated partly crystalline polymers, the amorphous regions of which have a lower conductivity than the crystalline regions. As a result, a charge is piled up at the crystal boundaries, in order to preserve current continuity. The TSD of heterogeneous systems is outlined by considering a charged short-circuited two-layer capacitor. ${ }^{* *}$

Denoting the layers by 1 and 2 , the pertinent field and current quations are

$$
\begin{gathered}
E_{1} l_{1}+E_{2} l_{2}=0 \\
j=\varepsilon_{0} \varepsilon_{1} \dot{E}_{1}+\gamma_{1} E_{1}=\varepsilon_{0} \varepsilon_{2} \dot{E}_{2}+\gamma_{2} E_{2}
\end{gathered}
$$

where $\gamma$ is the conductivity. In eq 28 we disregarded the small terms due to the currents $\varepsilon_{0} \dot{\varepsilon}_{1,2} E_{1,2}$. Now $\varepsilon$ and $\gamma$ change because of the heating. Hence, the equations lead to a differential equation for $E_{1}$

$$
\mathrm{d} E_{1} / \mathrm{d} t+\beta E_{1}=0
$$

** Less ideal systems will be considered in ref 34 . 
in which $\beta$ is variable, actually $\beta=\left(\gamma_{1} / l_{1}+\gamma_{2} / l_{2}\right) /$ $\varepsilon_{0}\left(\varepsilon_{1} / l_{1}+\varepsilon_{2} / l_{2}\right)$.

The solution of eq 29 reads

$$
E_{1}=E_{0} \exp \left(-\beta_{r} \eta\right)
$$

where $E_{0}$ is the initial field, and $\eta$ the reduced time: $\eta=S \int_{T_{R}}^{T} b_{T} \mathrm{~d} T . \quad E_{0}$ depends on the formation conditions. For a complete charging: $E_{0}=\sigma_{0} / C l_{1}$, where $\sigma_{0}$ designates the charge density at the boundary, and $C$ is the total capacity. The shift factor $b_{T}$ notably contains the temperature dependences of $\gamma_{1,2} ; \varepsilon_{1,2}$ are nearly constant. The conductivity usually increases according to an Arrhenius law.

Finally, eq 28 and 30 yield for the TSD current density

$$
j=\frac{\sigma_{0} \gamma_{1} l_{2}\left(\varepsilon_{2} / 1-\gamma_{2} / \gamma_{1}\right)}{\varepsilon_{0} \varepsilon_{1} l_{1}\left(\varepsilon_{2} / \varepsilon_{1}+l_{2} / l_{1}\right)^{2}} \exp \left(-\beta_{r} \eta\right)
$$

Although the current depends on two varying quantities $\gamma_{1,2}$, it can be shown that only one TSD peak appears. Note that the current may reverse during a heating. ${ }^{11}$ In practice, however, this will seldom occur.

Summarizing we have shown that the TSD of dipoles, as well as that of real charges, leads to characteristic thermograms. In fact, both mechanisms give rise to distinct peaks, as we shall see in the DISCUSSION OF EXPERIMENTAL RESULTS.

\section{The Relation of TDS to Conventional Measure- ments}

In view of the above theories, and anticipating the experimental results reported in the DISCUSSION OF EXPERIMENTAL RESULTS, we may state that TSD thermograms reflect the molecular relaxations of a polymer. Relaxation phenomena can also be studied with other techniques. Two well-established methods are measurement of the storage and loss moduli, and measurement of the dielectric constant and loss factor. ${ }^{37-41}$ These conventional methods are performed either isothermally or isochronously, while in TSD time and temperature are varied simultaneously.

In TSD of Metallized Electrets we showed that TSD becomes equivalent to isothermal measurements if instead of $t$ one takes the reduced time $\xi$. Thus, the temperature shift $a_{T}$ being known, it is rather simple to compare
TSD results with isothermal discharge data.

To calculate the equivalent time for an Arrhenius shift, we truncate the expansion of $E_{1}(x)$ after the first term, see eq 11. For a Debije relation we then find, according to eq 10.

$$
j=\alpha \Pi_{0} \exp \left\{-\left(\alpha s k T^{2} / A\right)\left(1-T_{R}{ }^{2} \alpha_{R} / T^{2} \alpha\right)\right\}
$$

whereas an isothermal discharge would produce a current density

$$
j=\alpha \Pi_{0} \exp (-\alpha t)
$$

Obviously, the equivalent time of TSD data equals

$$
t_{e}=\left(s k T^{2} / A\right)\left(1-T_{R}{ }^{2} \alpha_{R} / T^{2} \alpha\right) \simeq s k T^{2} / A
$$

for $s=1 \mathrm{~min} /{ }^{\circ} \mathrm{C}, t_{e}$ is found to be $2500 \mathrm{sec}$, and $150 \mathrm{sec}$ for side group and main chain relaxations respectively, of amorphous polymers. Since mechanical and dielectric relation are closely related, ${ }^{38-40}$ TSD may also be correlated to creep data.

Unfortunately, mechanical and dielectrical step-response data are scarce. We often have to be content with results from dynamic experiments. To compare TSD with isochronous observations, a time-frequency transformation has to be applied. This means that the dielectric loss $\varepsilon^{\prime \prime}(\omega)$ has to be calculated from the discharge current density $j(t)$. A pioneering review about this subject was published by Schwarzl and Struik. ${ }^{42}$ The exact relation between both quantities is given by a Fourier transform. However, the latter can only be evaluated approximately.

A first-order approximation for isothermal measurements was given by Hamon, ${ }^{43}$ see also Schwarzl. ${ }^{44}$ From the von Schweidler relation $j=j_{0} t^{-n}$, Hamon derived

$$
\varepsilon^{\prime \prime}(\omega) \sim j(t) / \omega \varepsilon_{0} E \quad \text { with } \omega t \sim 0.63
$$

where $E$ is the charging field. Assuming a thermorheologically simple behaviour, eq 35 also holds for TSD, provided that we replace $t$ by $t_{e}$. Obviously, TSD data are equivalent to $\varepsilon^{\prime \prime}$ data taken at an equivalent frequency

$$
\nu_{e} \sim 0.1 / t_{e}
$$

Since the interpretation of normal dielectrical and mechanical data is in an advanced state, these relationships provide powerful means for explaining TSD results. 
The equivalent frequency varies only slightly with temperature, so that TSD is nearly "isochronous". For the side-group and main chain relaxations of methacrylic polymers we find equivalent frequencies of about $10^{-4}$ and $10^{-3} \mathrm{~Hz}$, respectively. Due to these low frequencies the resolution of various relaxations is high, which makes TSD very attractive. Step-response measurements also show a high resolving power, but they are very time-consuming.

TSD is mathematically related to other nonisothermal methods, for instance the analysis of thermoluminescent and photoconductive glow curves, ${ }^{31,45}$ differential scanning calorimetry ${ }^{46}$ and thermogravimetric analysis. ${ }^{47}$ The classical descriptions of annealing and vitrification of glasses are also similar. ${ }^{48,49}$

\section{Evaluation of TSD Data}

Although the DISCUSSION OF EXPERIMENTAL RESULTS will deal mainly with the moleecular interpretation of TSD, it seems worthwhile to illustrate how TSD data can be evaluated. The aim of the evaluation is to find the temperature shift or activation energy, the relaxation frequency, the relaxation strength and the distribution function. The evaluation proceeds in a way similar to that used in ordinary dielectric measurements, or that used for thermoluminescent or photoconductive-glow curves.

To calculate the activation energy we can take the initial rise, or halfwidth, of the current graphs. Assuming a nondistributed polarization we obtain for an Arrhenius shift

$$
\begin{gathered}
\mathrm{d} \ln j / \mathrm{d} 1 / T=-A / k \quad \text { if } \quad \xi<1 \quad(37) \\
k T_{m} / A=1.9677-3.2602 h+1.2925 h^{2} \quad \text { if } \quad h<1
\end{gathered}
$$

$$
k T_{m} / A=-1.0330+1.0328 h \quad \text { if } \quad h>1
$$

where $h$ is the ratio of half-width temperature to maximum temperature, $h=T_{h} / T_{m}$. Eq 38 are accurate to within less than $1 \%$ for $0 \leq k T_{m} / A \leq$ 0.1. A more sophisticated method to find $A$, which involves the whole graph, is to use nomograms like that shown in Figure 4.

The location of the current maxmum is typical of the polymer in question. The maximum occurs at

$$
(\mathrm{d} \tau / \mathrm{d} T)_{m}=-s
$$

where $\tau$ is the relaxation time: $\tau=1 / \alpha$. For an Arrhenius shift, eq 39 leads to: $s k\left(\alpha T^{2} / A\right)_{m}=1$, while for a WLF shift the maximum is found close to $T_{g}$.

Obviously, the maximum shifts in temperature when $s$ is varied. Therefore, $A$ can also be found from this shift. In addition, the ratio of the current maxima, which is almost proportional to $s$, can be invoked to calculate $A$. Indexing the quantities at the current maximum for fast heating with $f$, and for slow heating with $s$, we have, for a heating ratio of 2.5

$$
\begin{gathered}
(k T / A)_{f}=1.8562-4.7865 t+2.9304 t^{2} \\
(k T / A)_{f}=1.9433-3.3644 c+1.4213 c^{2}
\end{gathered}
$$

where $t=T_{f} / T_{s}$ and $c=j_{f} / 2.5 j_{s}$. Both eq 40 and 41 hold for $0 \leq(k T / A)_{f} \leq 0.1$, with a maximum error of $0.3 \%$ and $0.9 \%$, respectively. The use of eq 40 and 41 is limited to low activation energies, otherwise the methods become too in accurate. They are therefore difficult to apply to most polymers.

According to eq 10, the ratio of the released current $j$ to the frozen-in charge $\Pi$ determines $\alpha$ and, indirectly, $a_{T}$. Unfortunately, this straightforward formula cannot be applied directly, for integration of the measured current yields the released charge, and not the frozen-in charge. However,

$$
\int_{0}^{t} j d t=\Pi_{0}-\Pi
$$

where $\Pi_{0}$ is the initial frozen-in charge: $\Pi_{0}=\int_{0}^{\infty} j d t$. The latter integral can only be evaluated accurately for non-overlapping peaks. From $\Pi_{0}$ one easily finds the relaxation strength $\varepsilon_{s}-\varepsilon_{\infty}$. For full charging we have

$$
\varepsilon_{s}-\varepsilon_{\infty}=\Pi_{0} / \varepsilon_{0} E
$$

The integral eq 42 was computed by means of Simpson's rule.

The current thermograms are frequently broader than the nomograms of Figure 4 because there is a distribution of relaxation frequencies. To find $f(\alpha)$, the integral eq 14 has to be solved. In viscoelastic work, Staverman and Schwarzi, ${ }^{30}$ this is often done by taking $\exp \left(-\alpha_{r} \xi\right)=1$ for $\left(\alpha_{r} \xi\right)_{0} \leq 1$, and $\exp \left(-\alpha_{r} \xi\right)=0$ 


\section{J. van TuRnhout}

for $\left(\alpha_{r} \xi\right)_{0} \geq 1$.

Assuming that the distribution arises from the preexponential factor, this approximation reduces eq 14 to

$$
\Pi / \varepsilon_{0} E=\left(\varepsilon_{s}-\varepsilon_{\infty}\right) \int_{\xi_{0}}^{\infty} F f\left(\tau_{r}\right) \mathrm{d} \tau_{r}
$$

In which we have changed from $\alpha_{r}$ to the more commonly used $\tau_{r}$. By differentiating eq 44 we obtain $f\left(\tau_{r}\right)$ explicitly, for a complete charging, i.e., $F=1$, we have

$$
f\left(\tau_{r}\right)=-\Pi_{0}^{-1}(\mathrm{~d} \Pi / \mathrm{d} \xi)_{0}=\Pi_{0}^{-1}\left(j / a_{T}\right)_{0}
$$

where $\tau_{r}=(\xi)_{0}{ }^{-1}$. For an Arrhenius shift $a_{T}$ can be eliminated by substituting $a_{T}=(A / s k)\left(\tau_{r} / T^{2}\right)_{0}$, which gives

$$
\tau_{r} f\left(\tau_{r}\right)=L\left(\tau_{r}\right) \sim\left(s k / \Pi_{0} A\right)\left(j T^{2}\right)_{0}
$$

In eq $46, L\left(\tau_{r}\right)$ designates the logarithmic distribution function, which, apart from a constant, can be found simply by multiplying $j$ by $T^{2}$.

Likewise, there may be a distribution for the activation energy. According to Gross ${ }^{10}$ this is even more likely, for such a distribution, eq 9 takes the form

$$
\Pi / \varepsilon_{0} E=\left(\varepsilon_{s}-\varepsilon_{\infty}\right) \int_{0}^{\infty} F g(\boldsymbol{A}) \exp \left(-\alpha_{r} \xi\right) \mathrm{d} \boldsymbol{A}
$$

Using the same approximation method and taking $F=1, g(A)$ is found to be

$$
g\left(A_{0}\right)=-\Pi_{0}^{-1}(\mathrm{~d} \Pi / \mathrm{d} A)_{0}
$$

where $A_{0}$ is given by

$$
\left(\alpha_{r} \xi\right)_{0}=1 \text { or } A_{0} \exp \left(A_{0} / k T\right)=s \alpha_{r} k T^{2}
$$

Changing the differentiation to $T$, and neglecting terms in $k T / A_{0}$, one obtains

$$
g\left(A_{0}\right)=s j / k \Pi_{0}\left(2+A_{0} / k T\right)
$$

This is again a simple formula. Apparently, TSD is also suitable for finding distribution functions. Equations similar to eq 48 and 49 were derived by Vand and Primak, ${ }^{50,51}$ for the evaluation of annealing experiments.

\section{TSD Using Air Gaps}

The isothermal discharge involving air gaps was discussed in ref. ${ }^{20}$ In this case we have a short-circuited three-layer capacitor, in which the field within the polymer differs from zero. Consequently, the conduction current flowing through the sample produces an external displacement current. The fact that this technique allows the actual detection of real-charge decay makes it well-suited for studying homocharging with non-contacting formation electrodes. An additional advantage is that if desired the persisting charge can be determined directly.

Referring to ref 20 , we summarize the pertinent equations briefly. In general, a polarization $\Pi$, a volume charge $\rho$ and surface charges $\sigma_{1,2}$ will be present. Their effective surface density on side 1 of the electret amounts to

$$
q_{1}=\sigma_{1}+\int_{0}^{l} \rho \mathrm{d} x-l^{-1} \int_{0}^{l}(\Pi+\rho x) \mathrm{d} x
$$

We assumed $\Pi$ to be a heterocharge, and $\sigma_{1}$ and $\rho$ to be homocharges.

During TSD, the real charges mainly decay by Ohmic conduction of the electret, whereas the polarization disappears by the stimulated reorientation of the dipoles, and by Ohmic charge compensation. These mechanisms decrease the effective charges $q_{1,2}$ according to

$$
\mathrm{d} q_{1} / \mathrm{d} t=\mathrm{d} q_{2} / \mathrm{d} t=-(\gamma \bar{E}+\mathrm{d} P / \mathrm{d} t)
$$

where $\vec{E}$ is the mean value of the internal field and $P$ the actual polarization. In eq 51 , drift and diffusion of the charges were not accounted for. Moreover we assumed the voltage across the ammeter to be zero.

Field $\bar{E}$ is related to the effective charges and and the air gaps $g_{1,2}$

$$
\bar{E}=\left(q_{1}-q_{t} g_{2}\right) / \varepsilon_{0}\left(l+\varepsilon_{\infty} g\right)
$$

where $g=g_{1}+g_{2}$ and $q_{t}=q_{1}-q_{2}$. The depolarization, $\mathrm{d} P / \mathrm{d} t$, is given by eq 1 .

By eliminating $\bar{E}$, we obtain three simultaneous differential equations for $q_{1,2} P$, respectively. In these equations, the conductivity $\gamma$, as well as the relaxation frequency $\alpha$ vary, which implies that they can only be solved numerically.

However, in our experiment, the internal field was small, because we chose $\varepsilon_{\infty} g<l$. Eq 1 then simplifies to

$$
\mathrm{d} P / \mathrm{d} t=-\alpha P
$$

so that the differential equations are uncoupled and can be solved analytically. Incidentally, eq 53 also holds for $\varepsilon_{s}=\varepsilon_{\infty}$, i.e., for lossless 
dielectrics. Since $\alpha$ varies, the solution of eq 53 becomes

$$
P=\Pi_{0} \exp \left(-\alpha_{r} \xi\right)
$$

By substituting eq 54 and 52 into 51 , we find for the charge and current density during TSD

$$
q=\exp \left(-\beta_{r} \eta\right)\left\{q_{0}-\Pi_{0} \alpha_{r} \int_{\xi_{R}}^{\xi} \exp \left(\beta_{r} \eta-\alpha_{r} \xi\right) \mathrm{d} \xi\right\}
$$

$$
j=\alpha \Pi_{0} \exp \left(-\alpha_{r} \xi\right)-\beta q
$$

where $q=q_{1,2}-q_{t} g_{2} / g, \quad \beta=\gamma g / \varepsilon_{0}\left(l+\varepsilon_{\propto} g\right)$ and, $\eta$ and $\xi$ are the reduced times of $\beta$ and $\alpha$ respectively

$$
\eta=s \int_{T_{R}}^{T} b_{T} \mathrm{~d} T \quad \text { and } \quad \xi=s \int_{T_{R}}^{T} a_{T} \mathrm{~d} T .
$$

By way of illustration, we calculated the TSD for a hetero- and homocharged electret. To this end we evaluated the integral of eq 55 numeri- cally, using Simpson's rule. We asumed that $\Pi_{0}>r_{0}$, where $r_{0}$ is the homocharged part of $q_{0}: q_{0}=r_{0}-\Pi_{0}$. We further supposed the homocharge to be the more stable, because Ohmic decay is delayed by short-circuiting. ${ }^{20}$ Figure 5 shows that the initial heterocharge reverses in sign and becomes an increasing homocharge which eventually drops to zero at high temperatures.

The evaluation of such thermograms is feasible only when the temperature dependences differ greatly, i.e., when $\eta<\xi$, in which case the decay of polarization and real charge can be separated, so that eq 55 reduces to

$$
q_{0}=r_{0} \exp \left(-\beta_{r} \eta\right)-\Pi_{0} \exp \left(-\alpha_{r} \xi\right)
$$

In practice the separation can be accomplished by combining the thermograms with those of metallized electrets. As an illustration, we decomposed the actual charge and current into

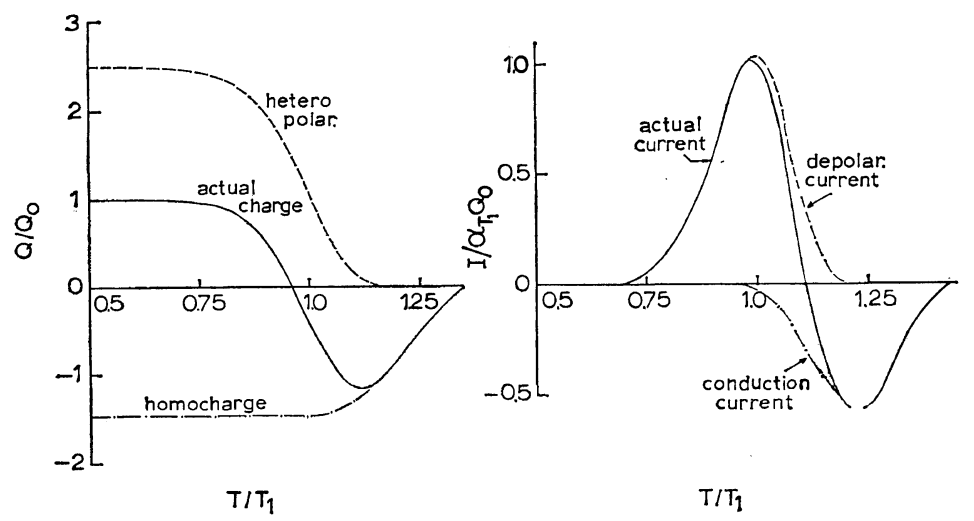

Figure 5. Calculated thermograms for TSD using air gaps. Indexing the maxima of the depolarization and conduction current with 1 and 2, respectively, we took: $A_{1} / k T=11.1, A_{2} / k T_{2}=11.4, T_{1} / T_{2}=0.82$ and $\Pi_{0}=2.45 Q_{0}$.

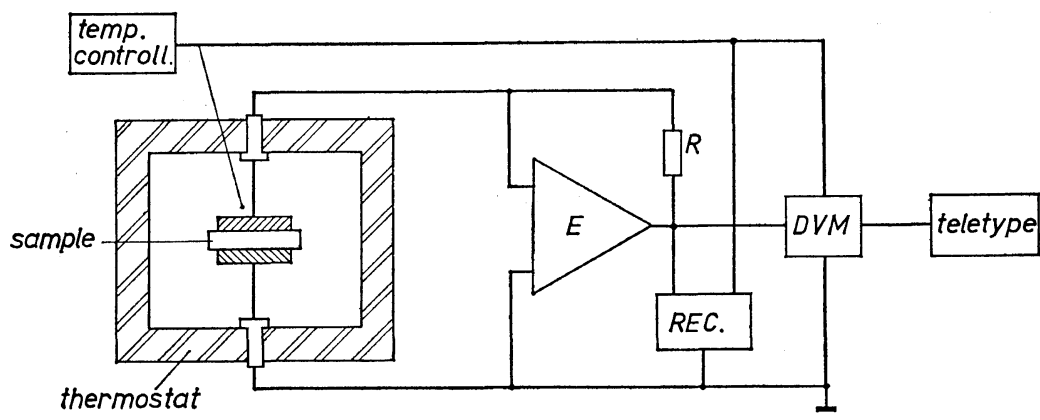

Figure 6. Set-up for automatic TSD measurements. 


\section{J. van Turnhout}

their components (Figure 5).

\section{THE EXPERIMENTAL SET-UP}

Figure 6 shows the equipment for TSD. The electret is placed in a thermostat, between two electrodes connected to a sensitive ammeter. The heating is achieved by circulating vaporized liquid nitrogen. The temperature is programmed by a motor-driven potentiometer and regulated by an electronic PID-controller. The temperature is sensed by a platinum resistor. Its resistance-temperature curve is linearized to ensure a constant heating rate for temperatures ranging from -180 to $250^{\circ} \mathrm{C}$. All TSD experiments were carried out with a heating rate of $1^{\circ} \mathrm{C} / \mathrm{min}$, unless stated otherwise.

The ammeter chosen was a stable vibrating capacitor electrometer E (Vibron 61 A, E. I. L. England), which was used in the feedback mode. Current as well as temperature was recorded and printed out. The results were also punched on a TELETYPE for evaluation by a digital computer.

Allowance has to be made for two instrumental errors. The first is due to the small voltage across the ammeter. This causes a part of the current to flow back through the sample. The part lost, $\delta j$, depends on the resistance ratio of electrometer and sample $R / R_{s}$

$$
\delta j=j R\left(R+R_{s}\right)^{-1}
$$

Obviously, for a correct measurement we must have $R \ll R_{s}$. This condition was satisfied, up to high temperatures, by putting $R$ in the feedback loop of $E$. $^{*}$

Moreover, many metallized polymers generate a parasite current well above the glass temperature. We attribute this to a small electrochemical potential difference, which may give rise to an appreciable current due to the increasing conduction of the polymer. The onset of this current can be ascertained by performing a TSD without charging. All our TSD thermograms will be given up to this onset.

\section{DISCUSSION OF EXPERIMENTAL RESULTS}

In this paragraph we confine ourselves to the molecular interpretation of TSD data. Details of their evaluation will be published elsewhere. ${ }^{34}$

Figure 7 shows results for two methacrylic polymers, viz., PEMA and PMMA. As depicted in the figure, both polymers have a polar ester

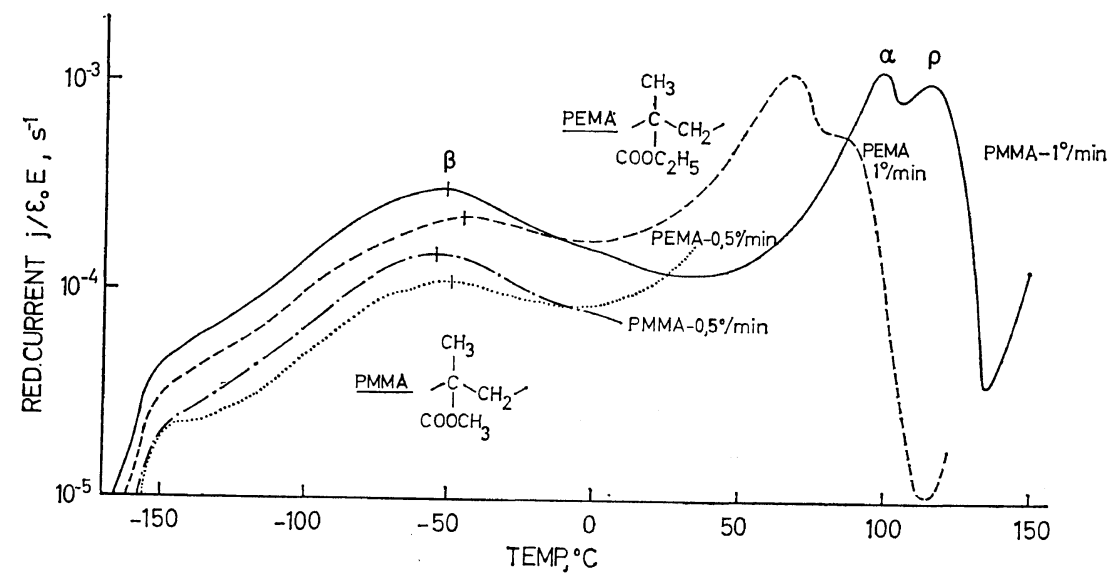

Figure 7. TSD thermograms of PEMA and PMMA. The temperature shift with heating rate is also shown.

* The current loss becomes larger when the current is also integrated to measure the charge released. We therefore abandoned electronic for numerical integration. 


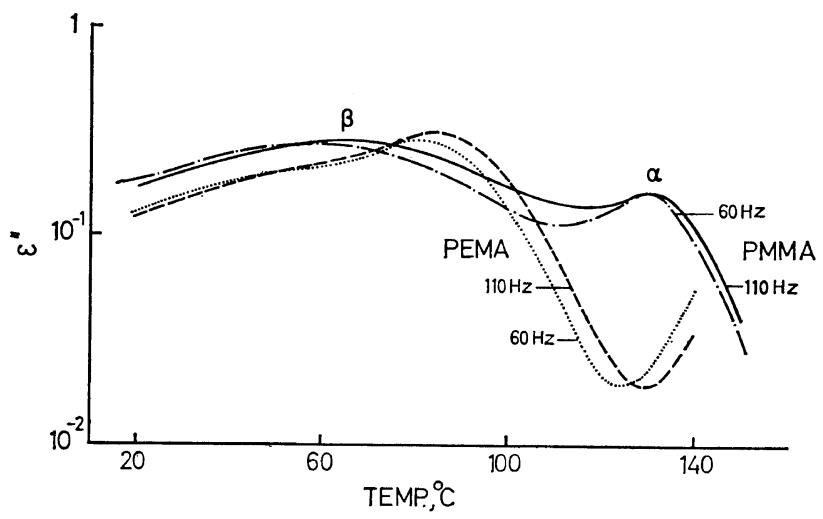

Figure 8. Dielectric loss factor for PEMA and PMMA at two frequencies. The shift of the $\beta$ peaks is the same as for TSD.

side group, -COOR, which can rotate with or without the main chain segments, $\mathrm{C}_{1} \mathrm{CH}_{2}$. Evidently, the cooperative motion of the bulky main chain and the side group requires more energy, whence it occurs at higher temperatures, at which the polymer becomes rubbery. To ensure that segmental motions, as well as local group-motions were frozen in, we cooled our specimens to $-180^{\circ} \mathrm{C}$.

The thermograms give the reduced current $v s$. the programmed temperature. For increasing temperatures we found three maxima, designated: $\beta, \alpha$, and $\rho$. For the $\rho$ and $\alpha$ peaks the current is highest. Following Heijboer's interpretation of conventional measurements, ${ }^{37,38}$ the $\beta$ peaks, which occur in the glassy state at $-45^{\circ} \mathrm{C}$ for PEMA and at $-51^{\circ} \mathrm{C}$ for PMMA $\left(s=1{ }^{\circ} \mathrm{C} / \mathrm{min}\right)$, are ascribed to the local motion of the polar side group. Because the ethyl substituent is larger and more sterically hindered, the ethyl $\beta$ peak is smaller than the methyl $\beta$ peak.

The $\alpha$ transitions, found at 66 and $102^{\circ} \mathrm{C}$ respectively, are due to the forced motion of the side groups together with the main chains. The larger ethyl groups push the main chains farther apart, thereby causing internal plastification. Consequently the ethyl $\alpha$ maximum is displaced to a lower temperature. Actually, the $\alpha$ peaks correspond, to the glass temperature $T_{g}$.

We attribute the $\rho$ peak, appearing at $85^{\circ} \mathrm{C}$ for PEMA and at $115^{\circ} \mathrm{C}$ for PMMA, to drifting of space charges. This peak increases with the conductivity of the polymer. Evidently, it originates from these particular conduction charges, which are trapped into the polymer during the formation. It is therefore sensitive to the electrode material and to extrinsic impurities such as water.

For both polymers, the charges accumulated will be nearly the same. Nevertheless, PMMA electrets will be more stable because of their higher $\alpha$ and $\rho$ temperatures.

As predicted by eq 39 and 40 , the peaks will shift with the heating rate. When heating is slow, the polymer responds sooner, giving a current maximum at a lower temperature. Its intensity is also lowered according to eq 41 , by about the ratio of the heating rates. Owing to their rather high activation energy of $1 \mathrm{eV}$, the $\beta$ peaks shift by only $4^{\circ} \mathrm{C}$. The shift of the $\alpha$ peaks, with activation energies of $5 \mathrm{eV}$, is hardly detectable and therefore not shown.

For comparison of our results with those of conventional measurements, dielectric data from Heijboer $^{38}$ are given in Figure 8. Only two peaks are present: $\alpha$ and $\beta$ both located above room temperature now. The $\rho$ peak, associated with the motion of real charges within the polymer, is absent. Due to the higher measuring frequencies, it occurs at such high temperatures that it merges into the conduction losses, ${ }^{*}$ which

* For TSD actual "conduction losses" are missing, because the internal field is zero. Its $\rho$ peak may only be masked by the conduction resulting from a small electrochemical potential difference. 


\section{J. van TURNHOUT}

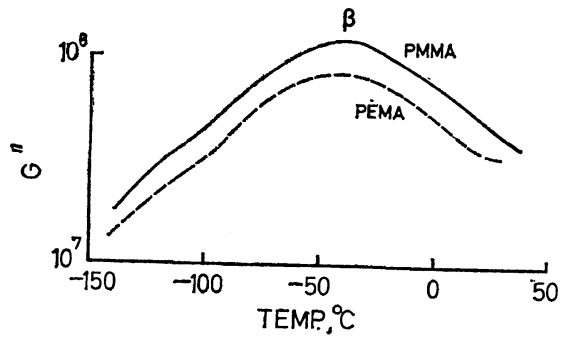

Figure 9. Loss modulus at $1 \mathrm{mHz}$ against temperature for PEMA and PMMA.

set in at $120^{\circ} \mathrm{C}$ for PEMA. Moreover, especially for PEMA, the $\alpha$ and $\beta$ peaks are not well-resolved. On account of its lower activation energy, the $\beta$ peak overtakes the $\alpha$ peak at the frequencies given. This again indicates that TSD provides results at a much lower frequency.

This was affirmed by torsional creep experiments. ${ }^{52}$ The data presented in Figure 9 were obtained by Ir. J. Heijboer and Ir. L. C. E. Struik* of our institute. By a time-frequency transformation $^{44}$ they were converted to $1 \mathrm{mHz}$. As may be calculated from eq 36 , both $\beta$ peaks are located somewhat higher, viz., at $-43^{\circ} \mathrm{C}$ for PEMA and at $-45^{\circ} \mathrm{C}$ for PMMA, than we found for a heating rate of $1^{\circ} \mathrm{C} / \mathrm{min}$.

Reconsidering Figure 7 and 8 , we note that dielectrically the $\beta$ peak is larger than the $\alpha$ peak for PMMA, whereas we found the opposite. This discrepancy results from the different ways in which the molecular relaxations were probed. It can easily be verified from eq 35 and 36 that the increase of TSD $\alpha$ peaks with respect to $\beta$ peaks is caused by

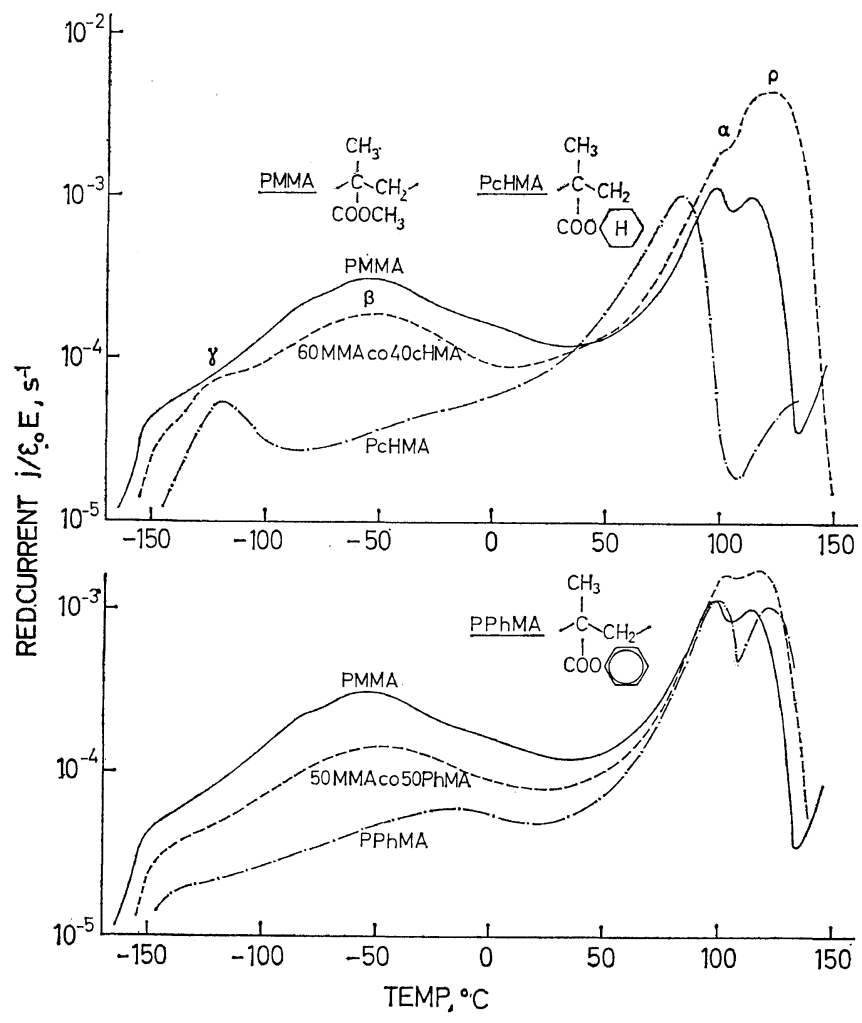

Figure 10. TSD of methacrylic homo- and coloplymers with cyclic alkyl side groups.

* The author wishes to thank his colleagues for making available these data prior to publication. 


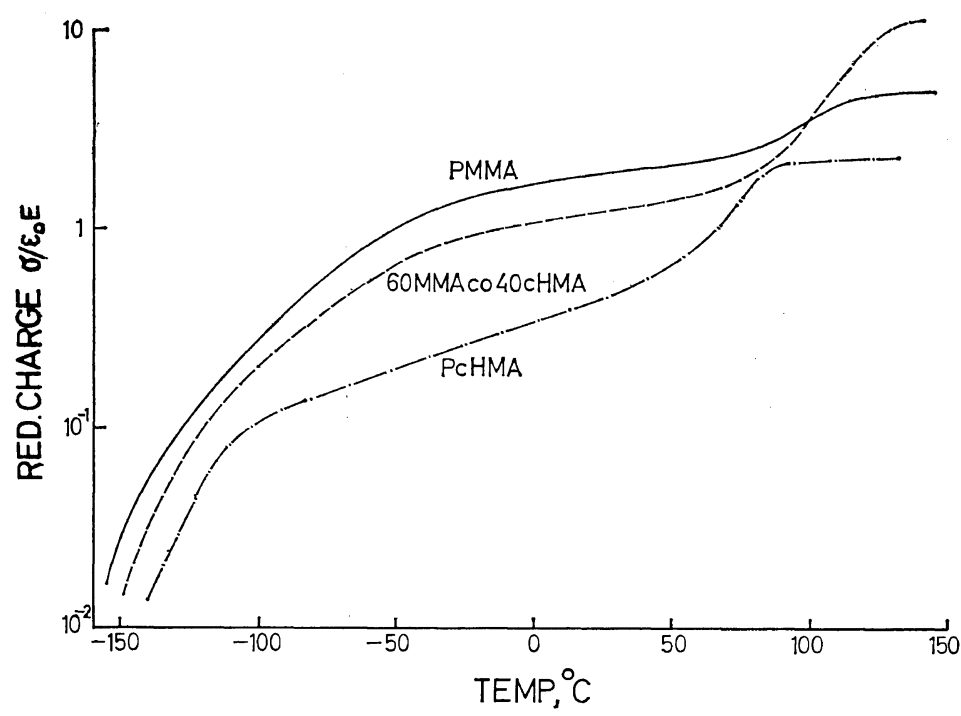

Figure 11. Released heterocharge during TSD of some methacrylic polymers.

their higher activation energies and temperatures.

Figure 10 shows results for methacrylic polymers with cyclic substituents: a flexible cyclohexyl group and a rigid phenyl group. In addition, two copolymers with MMA were investigated. For PcHMA an interesting $\gamma$ peak appears at $-118^{\circ} \mathrm{C}$. This was first interpreted by Heijboer ${ }^{53}$ as being due to a chair-chair transition of the 6-ring. So it is a local relaxation within the alkyl group. A $\beta$ peak is hardly observed, as any sweeping motion of the bulky side group is hindered. The rigid phenyl group gives no intra-alkyl relaxation. It does give, however, a small $\beta$ peak at $-13^{\circ} \mathrm{C}$, which is partly due to its higher polarity.

The current of the copolymers is about equal to the sum of the weight averages of their components

$$
j=j_{1} x_{1}+j_{2} x_{2}
$$

This does not hold for the $\rho$ peaks, which occur at $122^{\circ} \mathrm{C}$ for $60 \mathrm{MMA}$ co $40 \mathrm{cHMA}$ and at $118^{\circ} \mathrm{C}$ for $50 \mathrm{MMA}$ co $50 \mathrm{PhMA}$. They turn out to be definitely larger than expected from the mixing-rule. This seems to be a general feature, confirming the fact that copolymers are more conductive than homopolymers and often suffer higher dielectric losses.

In Figure 11 the charge during TSD is plotted.
Table I. Reduced heterocharge for various polymer electrets

\begin{tabular}{lrlr}
\hline $\begin{array}{c}\text { Homo- } \\
\text { polymer }\end{array}$ & $\sigma / \varepsilon_{0} E$ & \multicolumn{1}{c}{ Copolymer } & $\sigma / \varepsilon_{0} E$ \\
\hline PcHMA & 1.8 & 80 MMA co 20 AN & 14.4 \\
PMMA & 4.0 & 70 MMA co 30 AN & \multicolumn{1}{l}{26} \\
PPhMA & 3.1 & 60 MMA co 40 tBMAm & 12.3 \\
PC- $n$ & 0.8 & 60 MMA co 40 cHMA & 9.9 \\
PET & 1.0 & 80 MMA co 20 diMit & 14.3 \\
PVC & 16.1 & 50 MMA co 50 PhMA & 4.3 \\
& & 60 MMA co 40 S & 13.8 \\
& & 80 S co 20 AN & 82 \\
\hline
\end{tabular}

Table II. Names of the polymers used

\begin{tabular}{ll}
\hline PAN & polyacrylonitrile \\
PtBMAm, & poly ( $t$-butylmethacrylamide) \\
PC- $n$, & polycarbonate (Makrofol- $n$ ) \\
PE, & polyethylene \\
PET, & poly (ethylene terephthalate) (Mylar) \\
PcHMA, & poly (cyclohexyl methacrylate) \\
PdiMIt, & poly (dimethyl itaconate) \\
PMMA, & poly (methyl methacrylate) \\
PPhMA, & poly (phenyl methacrylate) \\
PS, & polystyrene \\
PTFE, & polytetrafluoroethylene \\
PVC, & poly (vinyl chloride) \\
PVF $_{2}$, & poly (vinylidene fluoride) \\
\hline
\end{tabular}




\section{J. van TURNHOUT}

It behaves less spectacularly, only having inflexion points corresponding to the $\rho, \alpha, \beta$, and $\gamma$ transitions. The final value of the reduced charge yields the relaxation strength $\varepsilon_{s}-\varepsilon_{\infty}$, see eq 43 . Note that the charge of the copolymer is distinctly higher.

In Table I the relaxation strength due to the $\alpha$ and $\rho$ peaks are compiled for various polymers (their abbreviations are explained in Table II). As expected, higher charges are obtained for more polar polymers. The values for the copolymers are strikingly high. Yet for good electrets, not only the charge matters. They should also possess good stability, for which a high $T_{g}$ and a low conductivity are prerequisites. Also in these respects, copolymers are promising, in particular MMA co cHMA and MMA co S.

Table III. Glass temperatures as found by TSD $\left({ }^{\circ} \mathrm{C}\right)$

\begin{tabular}{lrr}
\hline Polymer & $\begin{array}{c}\text { TSD } \\
\text { values }\end{array}$ & $\begin{array}{c}\text { Literature } \\
\text { value }\end{array}$ \\
\hline PcHMA & 83 & 90 \\
PEMA & 66 & 65 \\
PMMA & 106 & 105 \\
PPhMA & 105 & 105 \\
PET & 88 & 81 \\
PC- $n$ & 152 & 149 \\
89 TFE co 11 HFP & 75 & 77 \\
PVC & 69 & 68 \\
\hline
\end{tabular}

In Table III the location of some TSD $\alpha$ points are compared with dilatometric $T_{g}$ values cited in ref 40, 54. There is close agreement between them, which suggests that TSD is very suitable for determining the glass temperature of a polymer.

It is important to know the influence of the formation conditions on the thermograms. This is shown in Figure 12 for PET film (Mylar C, $25 \mu \mathrm{m}$, du Pont, U. S. A.). From top to bottom, the figures shows the effects of temperature, field, time, and cooling rate. As a reference we chose $130^{\circ}-100 \mathrm{kV} / \mathrm{cm}-1.5 \mathrm{hr}-1^{\circ} \mathrm{C} / \mathrm{min}$.

The temperature has the largest effect. If it is too low, the $\rho$ peak, normally located at $123^{\circ} \mathrm{C}$, does not emerge. The position of the peaks is independent of the formation temperature, which indicates that the $\rho$ peak is not an

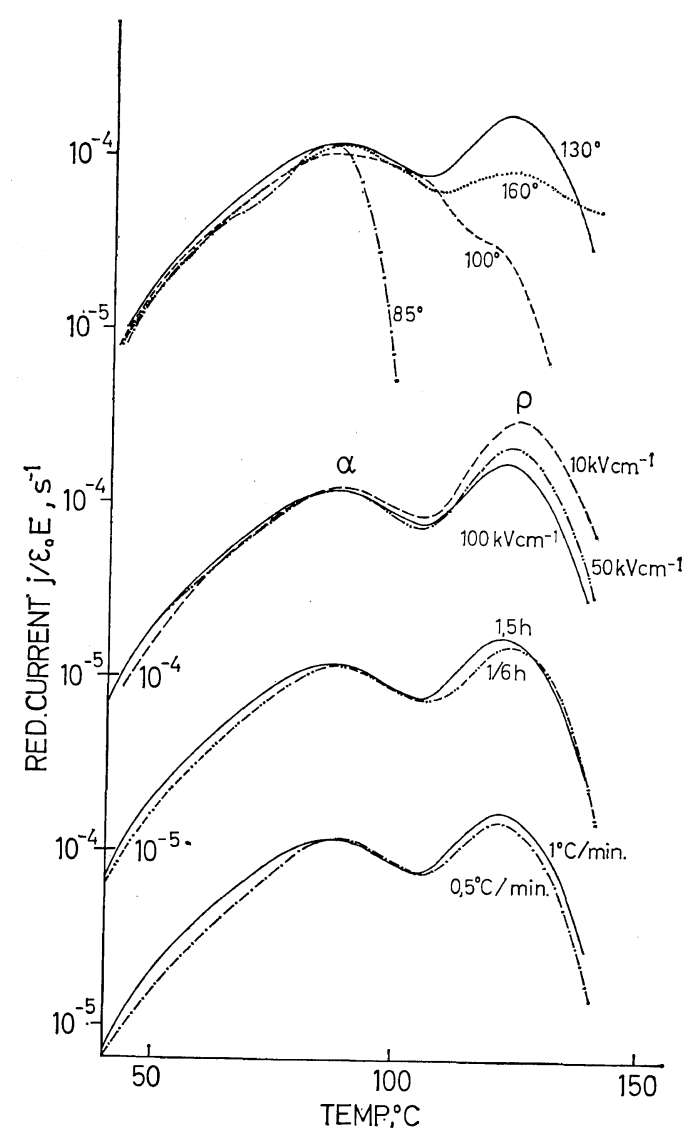

Figure 12. Results of PET electrets formed under different conditions; heating rate, $0.5^{\circ} \mathrm{C} / \mathrm{min}$.

artefact originating from charge exhaustion at the formation temperature. The $\alpha$ peak peak found at $87^{\circ} \mathrm{C}$ varies linearly with the field, while the space-charge peak behaves slightly nonlinearly. It is clear that the effective distribution function depends on temperature, because the $\alpha$ peak of the electret formed at $85^{\circ} \mathrm{C}$ is more or less cut off. The thermograms show no variation with formation time and cooling rate. In conclusion it may be said that to obtain a representative thermogram, the formation should be performed above $T_{g}$, the other conditions being less critical.

We still have to interpret the two peaks of PET. The $\alpha$ peak corresponds to the glassrubber transition of the amorphous part of the highly crystalline polymer. It arises from the cooperative motion of the glycol residue with 


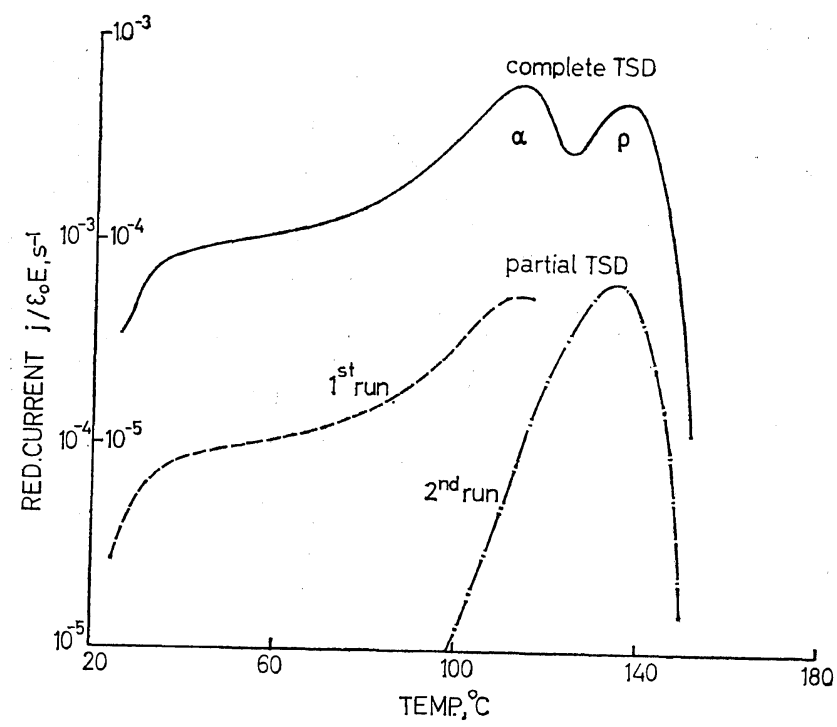

Figure 13. Isolating the $\rho$ peak of PMMA by partial TSD.

the COO dipoles of the main chain. The $\rho$ peak is due to space charges trapped on the crystal boundaries. These charges are released at a temperature at which the crystallization rate is highest. This characteristic temperature is known from differential thermal analysis as cold crystallization. Interestingly enough, it is not observed with normal dielectric or mechanical measurements.

Figure 13 illustrates an exceptional feature of TSD. We saw that although the $\alpha$ and $\beta$ peaks are well-resolved owing to the low frequency, the $\alpha$ and $\rho$ peaks, sometimes, are not. To study them separately we can either not charge the $\rho$ peak by choosing a low formation temperature, or clean the $\alpha$ peak by a partial TSD. ${ }^{9,55}$ The latter method is demonstrated for PMMA. After the first run-up to the $\alpha$ peak, we freeze in again and perform the second run to get the isolated $\rho$ peak.

Although TSD appears to be a powerful technique, some difïculties may arise in applying it. As already stated, the $\rho$ peak is sensitive to impurities. Figure 14 illustrates how absorbed water changes the thermogram noticeably. Thermal degradation of the polymer from annealing at too high temperatures also causes deviations.

These facts put in doubt the use of painted

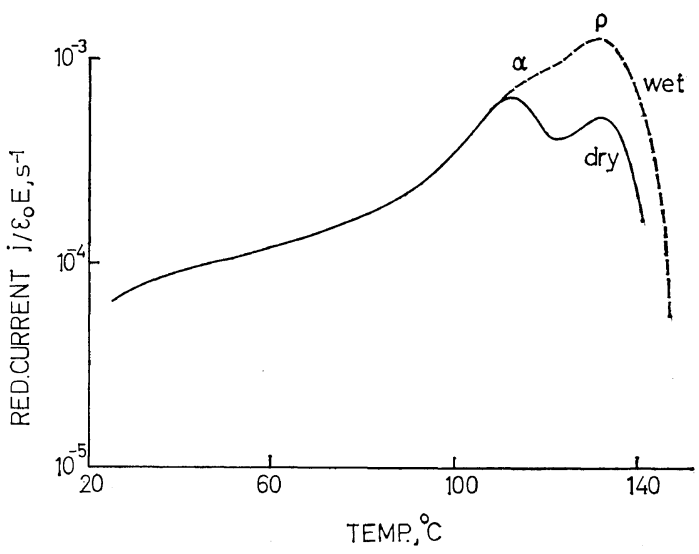

Figure 14. Influence of water on the TSD of PMMA. Before the formation the wet sample was held for $16 \mathrm{hr}$ at $70^{\circ} \mathrm{C}$ and $100 \%$ humidity.

electrodes for TSD, since most paints contain ethyl acetate, or similar solvents.

So far we have restricted ourselves to heterocharge decay. To study homocharge decay, air gaps were used by putting Teflon spacers of $0.5 \mathrm{~mm}$ between sample and electrodes. The combined hetero- and homocharge decay of a PMMA electret is shown in Figure 15. As expected, (see Figure 5) the resulting current and charge are lower than those given in Figures 13 and 14 . Since the homocharge is 


\section{J. van TURNHOUT}

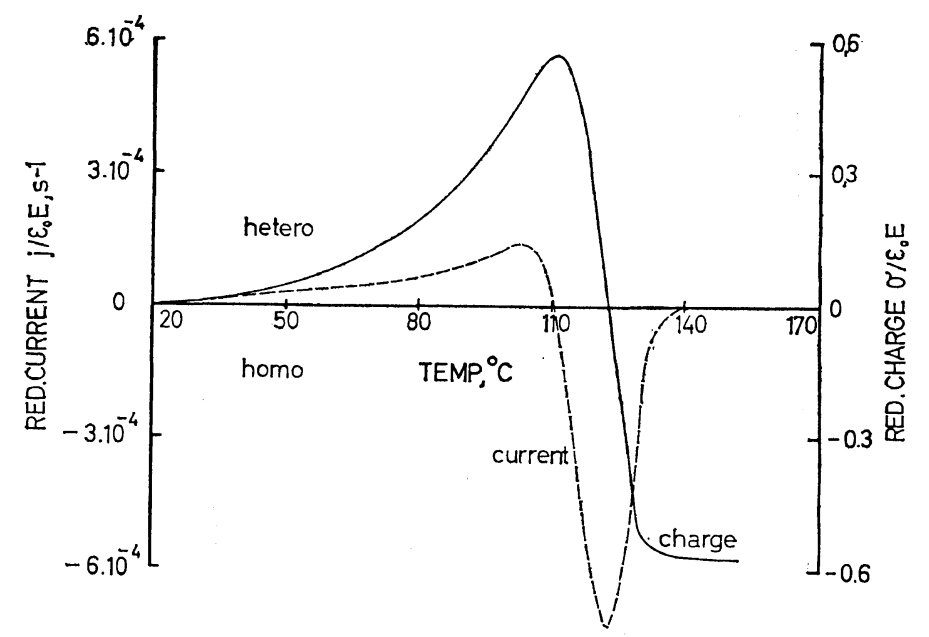

Figure 15. TSD of PMMA measured with air gaps. In contrast with Figure 5, the released charge and not the persisting charge, is shown.

larger and more stable, the current and charge become reversed in sign.

\section{CONCLUSIONS}

The present study of TSD partly elucidated the underlying molecular mechanisms of electrets, in particular of those operating during heterocharge. TSD turned out to be a powerful method for finding and developing better electret materials.

TSD, which possesses several features in common with DTA, can moreover be utilized for molecular spectroscopy of dielectrics. It has the advantage of a high resolving power, on account of the low frequencies involved. It allows the investigation of all polymer relaxations, including that of the glass-rubber transition. In fact, a new kind of relaxation, probably due to space charges was detected. This relaxation was particularly pronounced in copolymers, which were found to store higher charges than homopolymers.

TSD is recommended as an analyzing tool for research on electrostatics. Here, also, it may eventually reveal the molecular mechanisms involved.

Future work should be aimed at a better understanding of the TSD of space charges; besides the time-temperature dependence of the dipolar distribution function should be in- vestigated in more detail.

Acknowledgements. The author sincerely thanks Ir. J. Heijboer, Dr. F. R. Schwarzl, and Dr. P. H. Ong for valuable discussions and for reading the manuscript. He is indebted to Miss M. P. van Duijkeren for preparing most of the polymers, and to Messrs G. Roozendaal, P. J. Nederveen, and R. Nauta for technical assistance. He is also grateful to Mr. P. Léger for corrections in the English.

\section{REFERENCES}

1. V. A. Johnson, US Government Research Reports TR-1045 and TR-1074, Harry Diamond Laboratories, Washington D.C., 1962.

2. B. Gross, "Charge Storage in Solid Dielectrics", Elsevier Publ., Amsterdam, 1964.

3. J. Euler, "Neue Wege zur Stromerzeung", Akad. Verlag, Frankfurt, 1964 Chapter 4.

4. V. M. Fridkin and J. S. Zheludev, "Photoelectrets and the Electrographic Process", Consult. Bureau, New York, N. Y., 1961, Chapter 1.

5. G. Wiseman, "Research and Development Studies on Electrets", Univ. Kansas, 1955.

6. L. M. Baxt and M. M. Perlman Ed., "Electrets and Related Electrostatic Charge Phenomena", Symposium Series, Electrochemical Society, New York, N.Y., 1968.

7. G. M. Sessler and J. E. West, J. Acoust. Soc. Amer., 40, 1433-1440 (1966).

8. H. Frei and G. Groetzinger, Phys. Z., 37, 
$720-724$ (1936).

9. C. Bucci, R. Fieschi, and G. Guidi, Phys. Rev., 148, 816-823 (1966).

10. B. Gross, J. Electrochem. Soc., 115, 376-381 (1968).

B. Gross in "Electrophotography", Applied Optics Supplement 3, J. N. Howard Ed., American Institute of Physics, New York, N.Y., 1969, pp 176-179.

11. B. Gross, to be published, two lectures given at Delft, Report CL 235, TNO, Delft, 1970.

12. J. van Turnhout, Reports CL 39 and CL 70, 1967; Reports CL 4 and CL 18, 1968, TNO, Delft; J. van Turnhout in "Advances in Static Electricity”, Vol. 1, W. F. de Geest Ed., Auxilia, Brussels, 1971 (2nd paper 1st International Conference on Static Electricity, Vienna, May 1970).

13. R. A. Creswell and M. M. Perlman, J. Appl. Phys., 41, 2365-2375 (1970).

14. M. M. Perlman, to be published in J. Appl. Phys.*

15. M. L. Miller and J. R. Murray, J. Polym. Soc., Part $A-2$, 4, 697-704 (1966).

16. S. Mascarenhas, N. Januzzi, and C. Arguello, J. Electrochem. Soc., 115, 382-388 (1968).

17. T. Takamatsu and E. Fukada, Polymer J., 1, 101-106 (1970).

18. A. C. Lilly, R. M. Henderson, P. S. Sharp, and L. L. Stewart, J. Appl. Phys., 41, 2001-2014 (1970).

19. T. Nedetzka, M. Reichle, A. Mayer, and H. Vogel, J. Phys. Chem., 74, 2652-2666 (1970).

20. J. van Turnhout in "Advances in Static Electricity”, Vol. 1, W. F. de Geest Ed., Auxilia, Brussels, 1971 (1st paper 1st International Conference on Static Electricity, Viena, May, 1970).

21. B. Gross and R. J. De Moraes, J. Chem. Phys., 37, 710-713 (1962).

P. V. Murphy and B. Gross, J. Appl. Phys., 35, 171-174 (1964).

22. A. N. Gubkin and B. N. Matsonashvili, Soviet Phys. Solid State, 4, 878-884 (1962).

23. O. G. Altheim, Ann. Phys., 35, 417-430 (1939).

24. A. G. Zhdan, V. B. Sandomirskii, A. D. Ozhederov, Solid-State Electronics, 11, 505-508 (1968).

25. V. F. Zolotaryov, D. G. Semak, and D. V. Chepur, Phys. Status Solidi, 21, 437-442 (1967).

26. E. F. Haugh, J. Appl. Polym. Sci., 1, 144-149 (1959).

27. K. C. Rush, J. Macromol. Sci. Phys., B2,

* We thank Prof. Perlman for sending us the MSS of this papers prior to publication.
179-204 (1968).

28. P. B. Macedo and T. A. Litovitz, J. Chem. Phys., 42, 245-256 (1965).

29. E. Hastings, “Approximations for Digital Computers', Princeton University Press, Princeton, 1955, p 188.

30. A. J. Staverman and F. R. Schwarzl in "Die Physik der Hochpolymeren", Vol. 4, H. A. Stuart Ed., Springer Verlag, Berlin, 1956, pp 1 $-121$.

31. M. C. Driver and G. T. Wright, Proc. Phys. Soc., 81, 141-147 (1961).

32. G. A. Dussell and R. H. Bube, Phys. Rev., 155, 764-779 (1967).

33. J. Lindmayer, J. Appl. Phys., 36, 196-201 (1965).

34. J. van Turnhout, to be published.

35. L. K. Monteith and J. R. Hauser, J. Appl. Phys., 38, 5355-5365 (1967).

36. G. Jaffé and Z. Le May, J. Chem. Phys., 21, 920-928 (1967).

37. J. Heijboer, Brit. Polymer, 1, 3-14 (1969).

38. J. Heijboer, F. R. Schwarzl, and H. Thurn, in "Kunststoffe", Vol. 6, Nitsche and Wolf Ed., Springer Verlag, Berlin 1962, pp 363-404.

39. F. R. Schwarzl, in "Chemie und Technologie der Kunststoffe", Vol. 1, Staverman/Houwink, Ed. Springer Verlag, Berlin, 1963, pp 633-712.

40. N. G. McCrum, B. E. Read, and G. Williams, "Anelastic and Dielectric Effects in Polymeric Solids', Wiley, New York, N. Y., 1967.

41. Y. Ishida, J. Polym. Sci., Part A2, 7, 18351861 (19.69).

42. F. R. Schwarzl and L. C. E. Struik, Advan. Mol. Relaxation Processes, 1, 201-525 (1967-1968).

43. B. V. Hamon, Proc. Inst. Electr. Eng., 99, 151155 (Monogr. 27) (1952).

44. F. R. Schwarzi, Rheol. Acta, 8, 7-17 (1969).

45. J. T. Randall and M. H. F. Wilkins, Proc. Roy. Soc., A184, 366-407 (1945).

46. B. Wunderlich, D. M. Bodily, and M. H. Kaplan, J. Appl. Phys., 35, 95-102 (1964).

47. A. Broido, J. Polym. Sci., Part A-2, 7, 17611773 (1969).

48. W. L. Bragg and E. J. Williams, Proc. Roy. Soc., 145, 717-723 (1934).

49. A. Q. Tool, J. Amer. Ceram. Soc., 29, 240 253 (1946).

50. V. Vand, Proc. Phys. Soc., A55, 222-245 (1943).

51. W. Primak, J. Appl. Phys., 31, 1524-1533 (1960).

52. C. W. van der Wal and R. H. J. W. A. Drent, Rheol. Acta, 7, 265-271 (1968).

53. J. Heijboer, Kolloid-Z., 148, 36-47 (1956); ibid., 171, 7-15 (1960).

54. J. Brandup and E. M. Immergut, "Polymer Handbook', Interscience, New York, N.Y., 1966.

55. K. H. Nicholas and J. Woods, Brit. J. Appl., 15, 783-795 (1964). 